\title{
Acute Acalculous Cholecystitis
}

National Cancer Institute

\section{Source}

National Cancer Institute. Acute Acalculous Cholecystitis. NCI Thesaurus. Code C35579.

Acute inflammation of the gallbladder in the absence of gallstones 